\title{
The effect of vision on the perception of the noise produced by a chiller in a common living environment
}

\author{
Francesco Aletta $^{\text {a) }}$, Massimiliano Masullo ${ }^{\text {b) }}$, Luigi Maffei ${ }^{\text {()) }}$ and Jian Kang ${ }^{\text {d) }}$ \\ (Received: 12 November 2015; Revised: 17 February 2016; Accepted: 7 April 2016)
}

\begin{abstract}
Over the years, different noise sources related to community noise and their potential for eliciting noise annoyance have been investigated. Despite being a very common source within the urban realm, there is a current lack of literature on the perception of noise produced by small-medium sized sources like chillers and airconditioning devices. There are relatively few studies on the influence of audiovisual factors on noise perception for such sources. The main aim of this paper was to investigate the influence of the visibility of a chiller on perceived loudness and self-reported annoyance in a common indoor environment. Furthermore, this research aimed to investigate whether the abovementioned effects persisted in the presence of a cognitive task. To this purpose, two laboratory experiments were performed by means of an immersive virtual reality (IVR) system. Results show that the distance of the chiller influenced the noise perception, while the visibility of the chiller itself does not, assuming that a visual reference context is provided. Regarding the cognitive task, it was found to reduce the mean individual ratings of both the perceived loudness and noise annoyance related to the chiller, signifying that for the investigated sound source characterized by small spectral and temporal variation, it might have inhibited the attentional listening. These findings suggest that there is room for the implementation of recommendations for the design and management of such sources in the increasingly dense built environment. (C) 2016 Institute of Noise Control Engineering.
\end{abstract}

Primary subject classification: 63.2; Secondary subject classification: 51.6

\section{INTRODUCTION}

Most modern cities are facing an increasing densification of the urban realm. Such a process inevitably leads to an increasing exposure for urban housing residents to environmental stressors, like odors, noise and vibration. Community noise is indeed a major source of complaints and it is likely to elicit psycho-physiological stress responses; thus legislation frameworks provide that sound-pressure levels from community noise sources should not exceed specific thresholds ${ }^{1}$.

a) School of Architecture, University of Sheffield, Western Bank, S102TN, Sheffield, UNITED KINGDOM; email: f.aletta@sheffield.ac.uk.

b) Department of Architecture and Industrial Design, Second University of Naples, via San Lorenzo ad Septimum, 81031, Aversa, ITALY; email: massimiliano.masullo@unina2.it.

c) Department of Architecture and Industrial Design, Second University of Naples, via San Lorenzo ad Septimum, 81031, Aversa, ITALY; email: luigi.maffei@unina2.it.

d) School of Architecture, University of Sheffield, Western Bank, S102TN, Sheffield, UNITED KINGDOM; email: j.kang@sheffield.ac.uk.
Previous studies reported that, in home contexts, the intrusiveness of environmental noises could trigger adverse effects on wellbeing, even when the exposure occurs at relatively low levels ${ }^{2}$. One such adverse effect is noise annoyance, which is generally understood as a perceptual construct emerging from a negative individual assessment of the surrounding acoustic environment. A common definition provided by Guski et al. ${ }^{3}$ is that of a "multifaceted concept, covering mainly immediate behavioural noise-related effects and evaluative aspects".

Over the years, community noise and noise annoyance have been thoroughly investigated ${ }^{4,5}$, considering different noise sources, like road, railway and air traffic, wind farms and industrial plants ${ }^{6-9}$, but there is a current lack of literature on the perception of noise produced by mediumsized sources like chillers and air-conditioning devices. A study by Ballas ${ }^{10}$ introduced the concept of "ecological frequency" of environmental sounds. This chiefly relates to how often some environmental sounds are likely to be detected in everyday situations and contexts (i.e. home, work, school, travel, shopping, outdoor setting and street). The environmental sound with the highest ecological frequency was "air flow from an air-conditioning system" 
and many of the sounds with the highest frequencies reported by the participants involved in data collection referred to mechanical sounds of similar origin (e.g. ceiling fan rotating, heating $\mathrm{A} / \mathrm{C}$ fan motor, appliance running). Thus, chillers and air-conditioning devices can be considered as highly relevant environmental sound sources, despite having received limited research consideration, compared to other sources. The spread of these systems enhanced the indoor users' experience by providing better comfort conditions. In contrast, in highly populated urban contexts, the noise generated by these sources, is likely to propagate to the surrounding buildings. The noise generation mechanisms for chillers and air-conditioning devices have been thoroughly investigated ${ }^{11-14}$. Overall, it is the result of a combination of different mechanical and aerodynamic sources, such as the vibration of the compressor, the vibration of the electric motor and the noise caused by the fans, the latter being produced by acoustic generation in the air flow itself ${ }^{15}$. Previous research reported that chillers and air-conditioning systems were often claimed to be the cause of severe noise complaints ${ }^{16}$; however, not much attention has been given to the individual response to this kind of noise and its related noise annoyance ${ }^{17}$ and only recently some studies started to investigate potential associations between perceived sound quality of fan noises and their corresponding psychoacoustic parameters ${ }^{18}$.

As a main consequence of environmental sounds, noise annoyance is often related to the physical characteristics of the sound. Alayrac et al. ${ }^{19,20}$ investigated the noise annoyance caused by different types of "industrial noise" (of which chillers and air-conditioning systems could be considered a sub-set). They defined four main categories of industrial noise according to spectral features (i.e. broadband noises, low-frequency noises, lowfrequency noises with main component at $100 \mathrm{~Hz}$ and noises with spectral components in middle frequencies) and proposed different physical indicators to predict the corresponding noise annoyance on listeners. On the other hand, it is also acknowledged that noise annoyance might also be influenced by a number of perceptual effects, like those related to the meaning of sounds, the context and the audio-visual environment ${ }^{21}$.

Due to its numerous implications in everyday life, the interaction between audition and vision has always attracted the researchers' attention. So far, it has been approached from different perspectives and thoroughly investigated in several scientific disciplines such as neoscience, cognitive psychology ${ }^{22-30}$, environmental psychology $^{31,32}$ and psychoacoustics ${ }^{33}$.

However, despite the significant efforts that have been made to investigate the perceptual interactions between audition and vision, there are relatively few studies on the influence that the visibility of the sound source may have on the sound perception for environmental noise sources in complex everyday scenarios, and results are not always consistent. Aylor and Marks ${ }^{34}$ investigated the loudness of white noise perceived by a group of participants, attenuated by different noise barriers. They found that perceived loudness was lower when the barrier was only partially screening the sound source (i.e. visible sound source), while it was surprisingly higher when the sound source was totally screened (i.e. non-visible sound source). For this reason they speculated that when the sound source was totally screened, participants expected its loudness to decrease. Thus, they possibly overestimated the sound coming from behind the barriers, which they perceived to be already "loud". In a study about noise barriers for highways, Joynt and Kang ${ }^{35}$ investigated the effect of preconception and visual preference for the barriers' materials on their performance in mitigating noise. Considering concrete, timber, metal, metacrilate and green barriers, the authors observed that, despite providing the same noise attenuation, green barriers were considered to be less effective. In another study about noise barriers for railway infrastructures, Maffei et al. ${ }^{36}$ observed that when the barriers were transparent (i.e. visible sound source) the perceived loudness and noise annoyance were lower than when the barriers were opaque (i.e. invisible sound source), even if the provided attenuation was the same. Peris et al. ${ }^{37}$ investigated the impact of the visibility of a railway on vibration annoyance. They observed that, at the same vibration exposure level, the odds ratio of people being highly annoyed by vibration from the railway was more than 1.6 higher when the railway was visible, compared to when it was not. Bangjun et al. ${ }^{38}$ investigated the effect of the visibility of road traffic on the noise annoyance perceived in a city park and a school building. The authors concluded that under the same acoustic environment, when the noise source (road traffic) was visible, the self-reported noise annoyance was higher. In their study on the impact of visual factors on noise perception caused by wind turbines, Pedersen and Larsman ${ }^{9}$ observed that visibility of the sound source highly increased the corresponding noise annoyance, possibly due to the "perceptual contrast" between the "technological" character of the sound source (i.e. the wind turbines) and the background context (i.e. rural environments).

In summary, individual responses to noises produced by different sound sources are varied, depending on both acoustical and non-acoustical factors, and there is no clear consensus on whether visibility of the sound source itself actually increases or decreases noise annoyance. Considering that noise produced by chiller and air-conditioning systems is becoming an increasingly frequent source in urban contexts, this requires more in-depth research. Furthermore, it is likely to expect that the visibility of such sources could also play a role in holistic environmental appraisals. 
Therefore, the main aim of this paper was to investigate the influence of the visibility of a chiller on perceived loudness and self-reported annoyance, and whether such influence changes according to the distance between the chiller and the individual. Furthermore, this research aimed to investigate whether the abovementioned effects persisted in the presence of a cognitive task. To this purpose, two laboratory experiments were performed by means of an immersive virtual reality (IVR) system. The results provide new insights on how people in common residential environments are likely to cope with these kinds of stressor and suggest that there is room for the implementation of recommendations for the design and management of such sources in the increasingly dense built environment.

\section{METHODS}

The present study is made of two separate experiments. The same participants were invited to take part and the same experimental set up was used for both experimental sessions, the difference between the two being the inclusion of a cognitive task or not. Therefore, Secs. 2.1 to 2.4 are common to both experiments.

\subsection{Participants}

Twenty-six undergraduates and postgraduates at the Second University of Naples, 19 to 46 years old, took part in the experiment ( 10 women and 16 men, $M_{\text {age }}=28.4$ years, $\mathrm{SD}=6.4)$. All participants reported normal hearing and vision. Participants were not reimbursed for their time and took part voluntarily. The sample size was defined through an a priori computation ${ }^{39}$ to achieve a minimum power $(1-\beta)$ of $80 \%$, a probability of error $(\alpha)$ of $5 \%$ and a medium effect size $(f)$ of $0.25^{40}$.

\subsection{Auditory and Visual Stimuli}

The sound of an industrial air cooled chiller unit was recorded $1 \mathrm{~m}$ from the source with a binaural headset connected to a portable recording device (SQuadriga Head Acoustics ${ }^{\mathcal{C}}$ ). The chiller was located on the top roof of an office building in a scarcely urbanized area, as shown in Fig. 1. Possible reflections from surrounding facades and background noise during the recording were therefore minimized and considered to be negligible. A 20-second audio excerpt was extracted from the recordings dataset and considered for further analysis. The reason for selecting this excerpt was to achieve a reasonably stationary noise to be representative of the sound source under normal working conditions. Figure 2 shows the one-third octave bands spectrum of the considered audio excerpt that resulted in a $L_{\mathrm{Aeq}-20 \mathrm{~s}}$ of $78 \mathrm{~dB}$, after the left and right channels were averaged for the sake of convenience.
In order to account for different source-receiver relative positions, the abovementioned audio signal was propagated at 10, 20 and $30 \mathrm{~m}$ according to the general method of calculation for the attenuation of sound during propagation outdoors ${ }^{41}$, as reported in Fig. 3. Consequently, the three attenuated audio excerpts were obtained to be used as auditory stimuli for the experimental design. The 10 , 20 and $30 \mathrm{~m}$ audio excerpts resulted in a $L_{\text {Aeq-20s }}$ of 46 , 40 and $36 \mathrm{~dB}$, accordingly.

A three-dimensional graphic model of an imaginary context was prepared. The scene and the point of view were set in a common living room with a large open window, facing a peri-urban context, with spread buildings located randomly in a country side. In the outdoor scenario, an industrial building with a reproduction of the recorded chiller units was located at three different distances, corresponding to three different visual scenarios, as per the sound propagation distances: 10, 20 and $30 \mathrm{~m}$. A fourth scenario had neither industrial building nor chiller included in the field of view of the observer. Therefore, four visual scenarios were achieved, as shown in Fig. 4. The rationale for setting the point of view in a living room was that this circumstance was assumed to be representative of plausible contexts where the investigated sound sources are most likely to be experienced.

\subsection{Immersive Virtual Reality (IVR) Set-Up}

Recent advances in immersive virtual reality systems provided new operative tools to investigate human crossmodal perception, as in such environments it is possible to present integrated audio-visual stimuli, reaching a good level of realism ${ }^{29,30}$. Audio-visual interactions are likely to work differently in complex everyday life situations and virtual reality technologies allow the experimenter to control for such conditions. There is still no clear consensus about the ecological validity of individual responses collected under laboratory conditions by means of IVR systems. However, such techniques are increasingly used for studies and simulations of the built environment with satisfactory results (for a review, see Ref. 42). In a recent study by Maffei et al. ${ }^{43}$ two groups of participants were asked to provide individual responses about overall acoustic and visual quality of a real environment (on site data collection) and its reproduced version in immersive virtual reality (laboratory experiment data collection). No statistically significant differences were observed between the two groups for both auditory and visual stimuli, highlighting the ecological effectiveness of such tools.

The auditory stimuli were made to generalize the observed results for the investigated sound source. While reproducing an auralization perfectly consistent with the surrounding environment (e.g. introducing outdoor- 


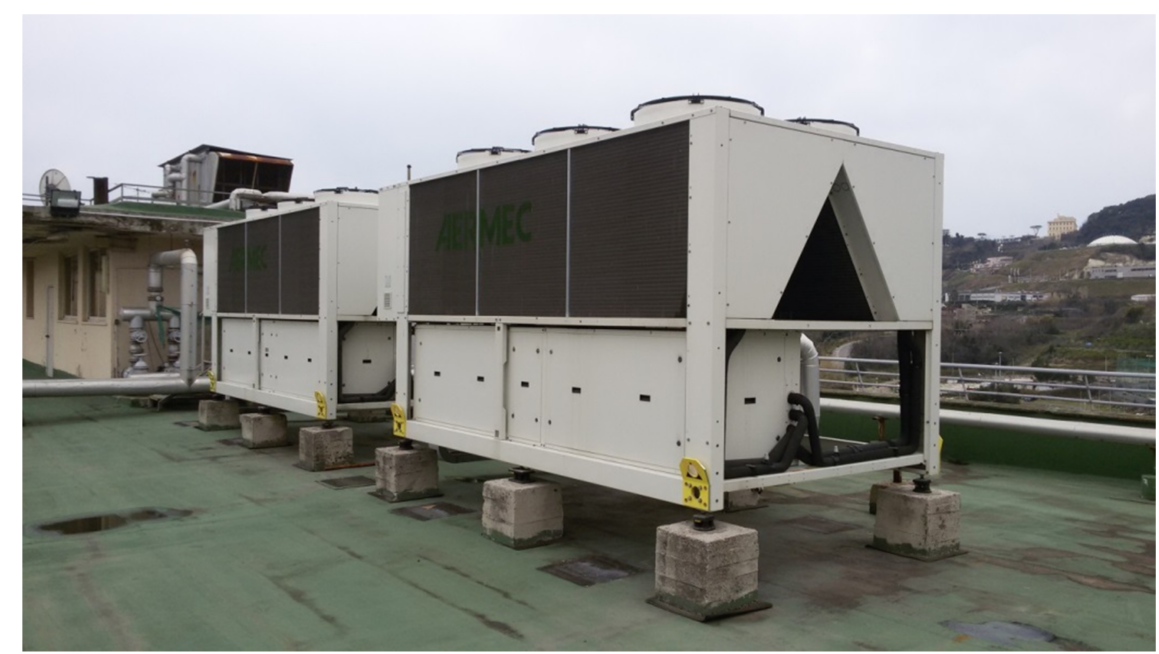

\section{Fig. 1-The chiller unit recorded for the audio stimuli of the experiment.}

to-indoor airborne noise reduction, or taking into account reflection and diffraction phenomena) would lead to results that were only valid for the investigated case (i.e. specific window size, room materials, etc.), it would also become case specific and therefore the auditory stimuli are less "realistic" in this way. The aim of this study is to test the effect of sound source visibility and how this can modify noise perception. The focus is less on whether people would perceive loudness in the virtual scenario as they would in the corresponding "real" one (i.e. absolute ratings), but rather that, given an auditory stimulus, people would consistently report differences between the visibility levels (i.e. relative ratings). This approach also corresponds to the substantially holistic methods recently adopted in literature, namely, to achieve a perceptually correct or "plausible" auralization rather than a physically correct one ${ }^{44-46}$, directly relating to participant's sensorimotor contingencies ${ }^{47}$, which is true overall for the proposed configuration.

Both the auditory and the visual stimuli previously described were uploaded to a Virtual Reality (VR) environment managed by dedicated software (WorldViz 4.0)

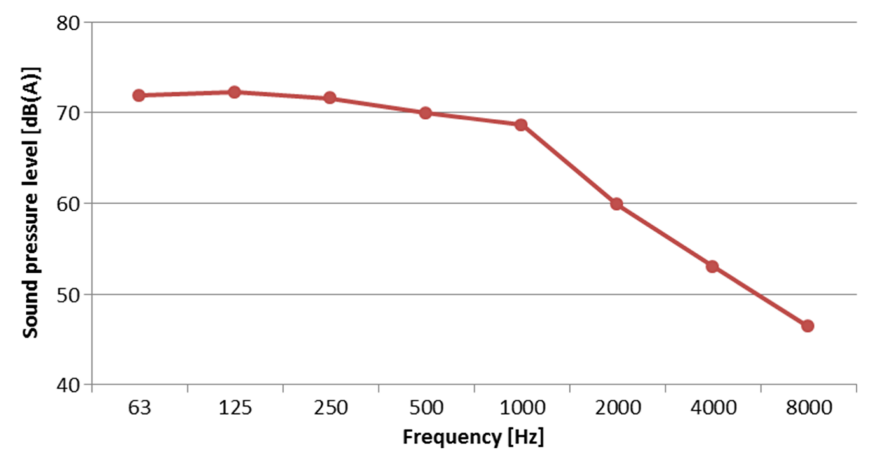

Fig. 2-One-third octave bands spectrum of the audio stimulus recorded on site, $1 \mathrm{~m}$ off the chiller (average of left and right channels). on a working station. The working station was connected to a combined playback system of headphones and subwoofer and to a visor for the representation of the visual scenarios. The headphones and the sub-woofer were calibrated by an artificial dummy head, in order to reproduce the same sound-pressure levels $( \pm 0.3 \mathrm{~dB})$ as recorded on site and the best fitting of the spectrum at the listener's ear. The reason for using a hybrid set-up (i.e. headphones and sub-woofer) was to compensate the headphones' limitations in properly reproducing the low-frequency contributions of the selected sound. The visual scenarios were presented through a system implemented with the eMagin Z800 3D Visor. The visor presented stereoscopic images at $800 \times 600$ resolution, refreshed at $60 \mathrm{~Hz}$, with a field of view of $40^{\circ}$. Real-time graphics were rendered by an Intel(R) Core(TM) i7 CPU $3.07 \mathrm{GHz}$ processor with an Nvidia GeForce GTX480 graphics card using the software WorldViz 4.0. The head's orientation and position were tracked using a six-degrees-of-freedom motion tracking system (Polhemus Patriot), as shown in Fig. 5 (binaural reproduction was not head-tracked).

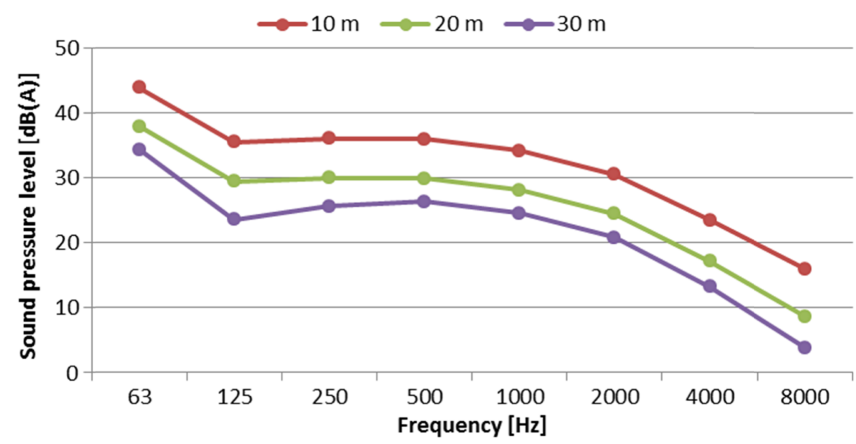

Fig. 3-One-third octave bands spectra of the three attenuated audio stimuli at 10, 20 and $30 \mathrm{~m}$ used for the experiments. 

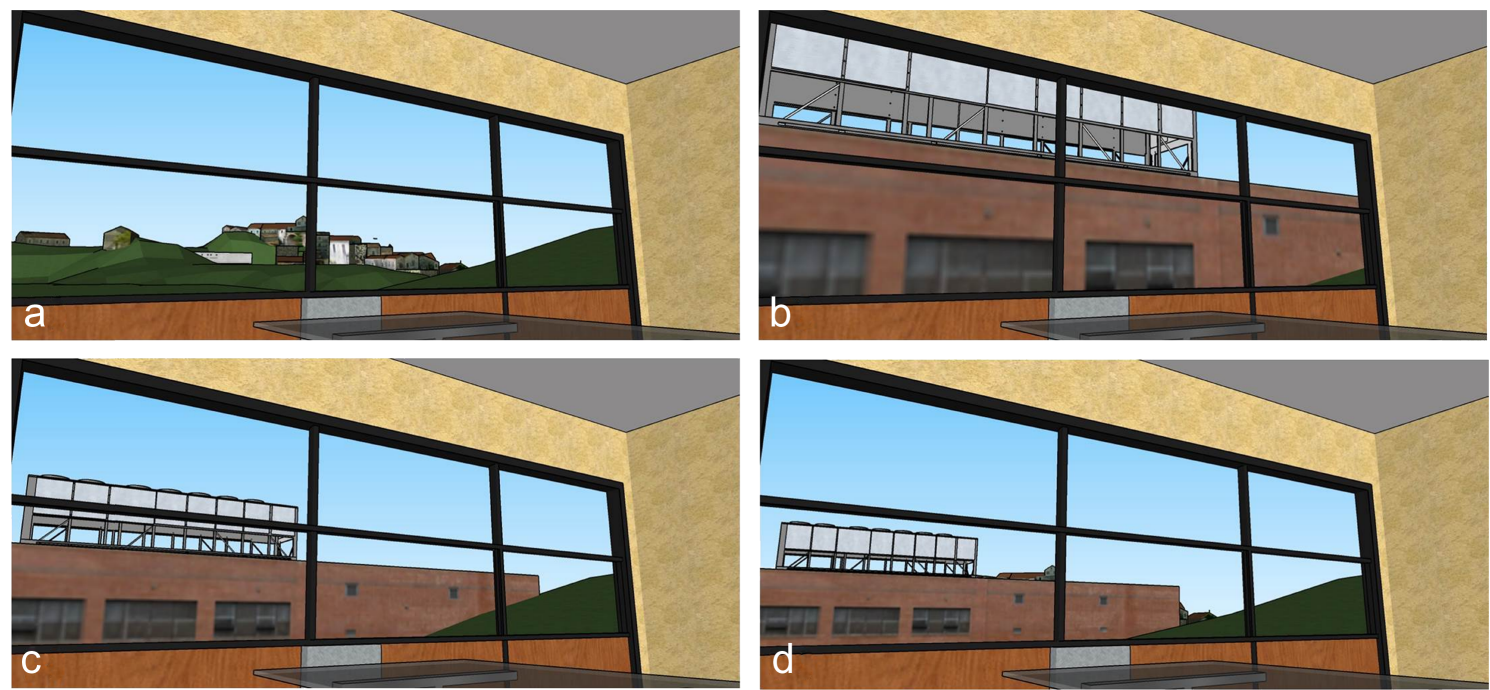

Fig. 4-Three-dimensional models of the visual scenarios: (a) No source, (b) $10 \mathrm{~m}$ source, (c) $20 \mathrm{~m}$ source and (d) $30 \mathrm{~m}$ source.

\subsection{General Experimental Design and Procedure}

Two factors were considered in this experiment: the distance factor and the visibility factor. The distance factor had three levels: $10 \mathrm{~m}, 20 \mathrm{~m}$ and $30 \mathrm{~m}$. These levels were representative of the three audio-visual scenarios with the chiller units at the three corresponding distances and noise levels. The visibility factor had three levels: "black screen," "visible chiller" and "invisible chiller," corresponding to the visibility condition of the sources producing the noise ("visible" and "invisible") plus an additional condition where no visual information was presented at all ("black screen"). Therefore, the design of

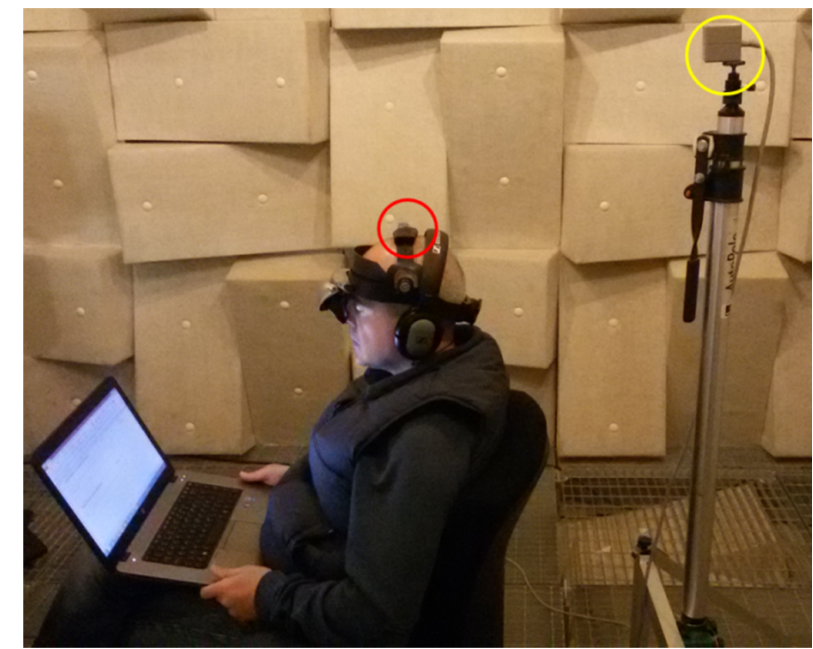

Fig. 5-A picture of the IVR experimental set-up with the head-mounted motion sensor (red circle) and the motion tracker (yellow circle). the experiment consisted of nine scenarios $(3 \times 3$ experimental conditions), as shown in Fig. 6.

The experiments were carried out in the anechoic room $(5 \times 5 \times 5 \mathrm{~m})$ of the Department of Architecture and Industrial Design of the Second University of Naples. Participants took part individually. Upon arriving, the participants were asked to sign the informed consent and then report if they had a normal or corrected to normal hearing and vision. Some demographic information was collected for descriptive purposes. Afterwards the experimenter read the instructions of the test. The participant was invited to sit on a chair in the middle of the room and he was given the IVR kit. Before the start of the series of visual and auditory stimuli, a training session was carried out, presenting a generic experimental scenario to let the participants familiarize with the virtual environment.

\subsection{Experiment 1: Perceived Loudness, Noise Annoyance and Visual Unpleasantness}

The nine conditions of the experimental design were presented in a randomized sequence for each participant. After each scenario participants were asked to answer two questions: (1) "How loud was the sound that you have just heard?" and (2) "How annoying was the sound that you have just heard?". For both questions, they could express their opinion on a 100-point scale by dragging a cursor between "not at all" (0) and "extremely" (100). When the scenarios where the chiller was visible were presented (i.e. first row in Fig. 6), an additional question was asked: (3) "How would you assess the visual presence of the sound source?". In this case participants could express their opinion on a 100-point scale by 


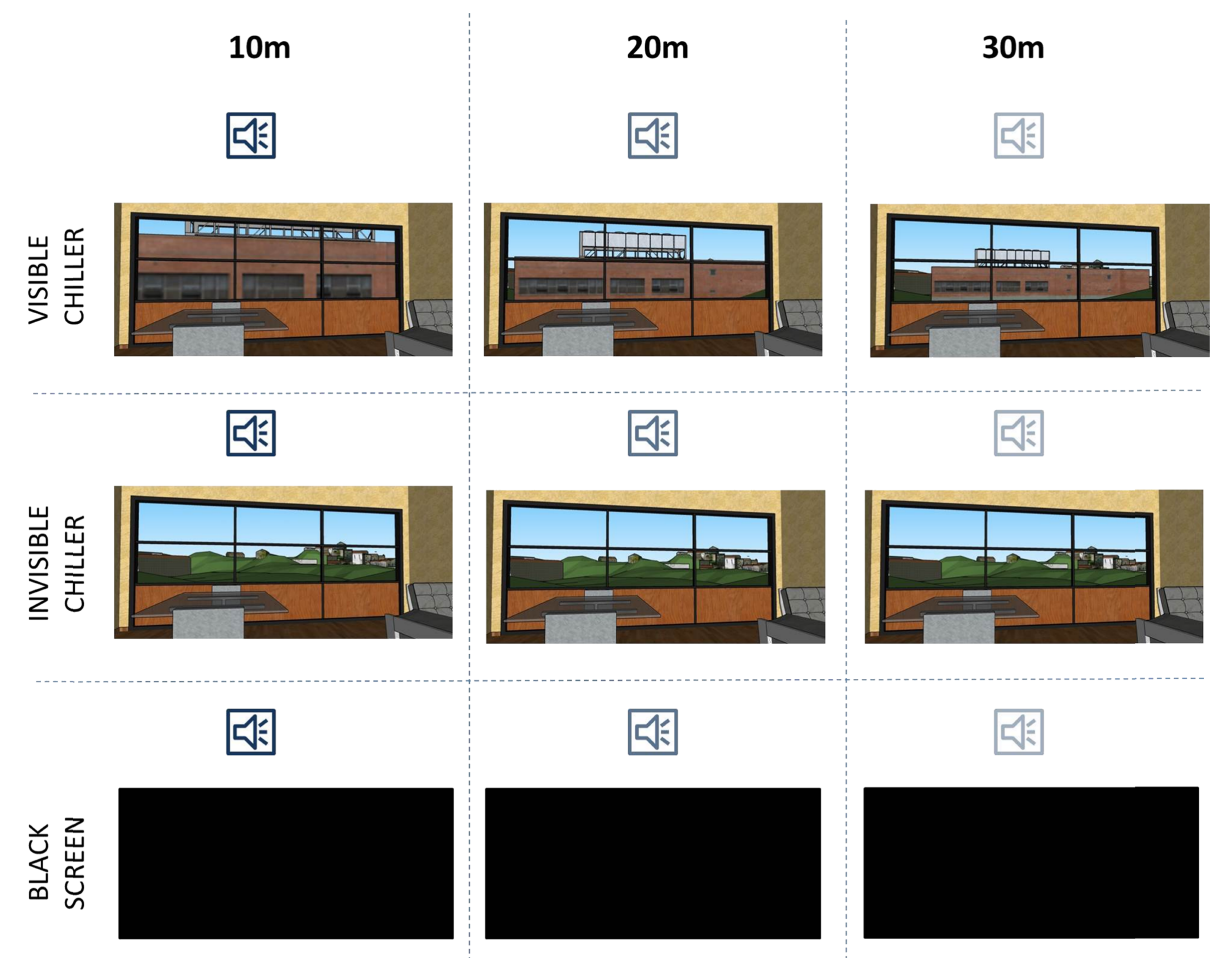

Fig. 6-Experimental design of the experiments 1 and 2.

dragging a cursor between "passable/neutral" (0) and "unpleasant/intolerable" (100).

\subsection{Experiment 2: Perceived Loudness, Noise Annoyance and Visual Unpleasantness with Cognitive Task}

The same experimental design was used in Experiment 2 as per in Experiment 1. In order to evaluate the effect of noise on an executive control process, participants were required to perform a cognitive task while experiencing the virtual scenarios. The selected task was "backward counting" for it requires high attentional resources and impacts executive functions ${ }^{48}$. For this task, participants were required to count backward in steps of seven starting from a given number. In this version of the task, 10 starting numbers randomly selected for each scenario and participant were considered. Each starting number was presented superimposed on the visual scenario via the visor.

The nine conditions of the experimental design were presented in a randomized sequence for each participant and participants were required to answer the same questions as per Experiment 1. Additionally, a control condition was considered where participants had to perform the cognitive task without any auditory or visual stimulus. The number of correctly generated numbers by the participants for each scenario was reported by the experimenter and further considered as a measure of cognitive performance $^{48}$.

\section{RESULTS}

\subsection{Experiment 1: Perceived Loudness, Noise Annoyance and Visual Unpleasantness}

A two-way repeated measures ANOVA was carried out to investigate the main effects and interactions of the distance and the visibility factors on the perceived loudness and the noise annoyance variable.

Results showed that the perceived loudness was affected by both the distance factor, $F(2,24)=29.172$, $p<.001$, partial $\eta^{2}=1.000$ and the visibility factor, $F(2,24)=5.580, p=.010$, partial $\eta^{2}=.808$. Post hoc analyses employing Bonferroni correction revealed that all differences in the perceived loudness ratings for the distance factor were significant: the 10-meter scenarios $(M=54.167)$ were perceived to be louder than the 20meter scenarios $(M=42.013)$, that were in turn louder than the 30 -meter scenarios $(M=38.000)$. Regarding the visibility factor, the scenarios with the black screen $(M=50.244)$ were perceived to be significantly louder than both the visible chiller scenarios $(M=43.603)$ and the invisible chiller scenarios $(M=40.333)$. However, no significant difference was observed between the visible and invisible chiller scenarios, or any interaction between the distance and visibility factors. Figure 7 reports the estimated marginal means for the loudness variable, comparing the visibility levels, as a function of distance. Figure 8 shows the individual assessments of perceived loudness for the visibility levels, as a function of distance. 


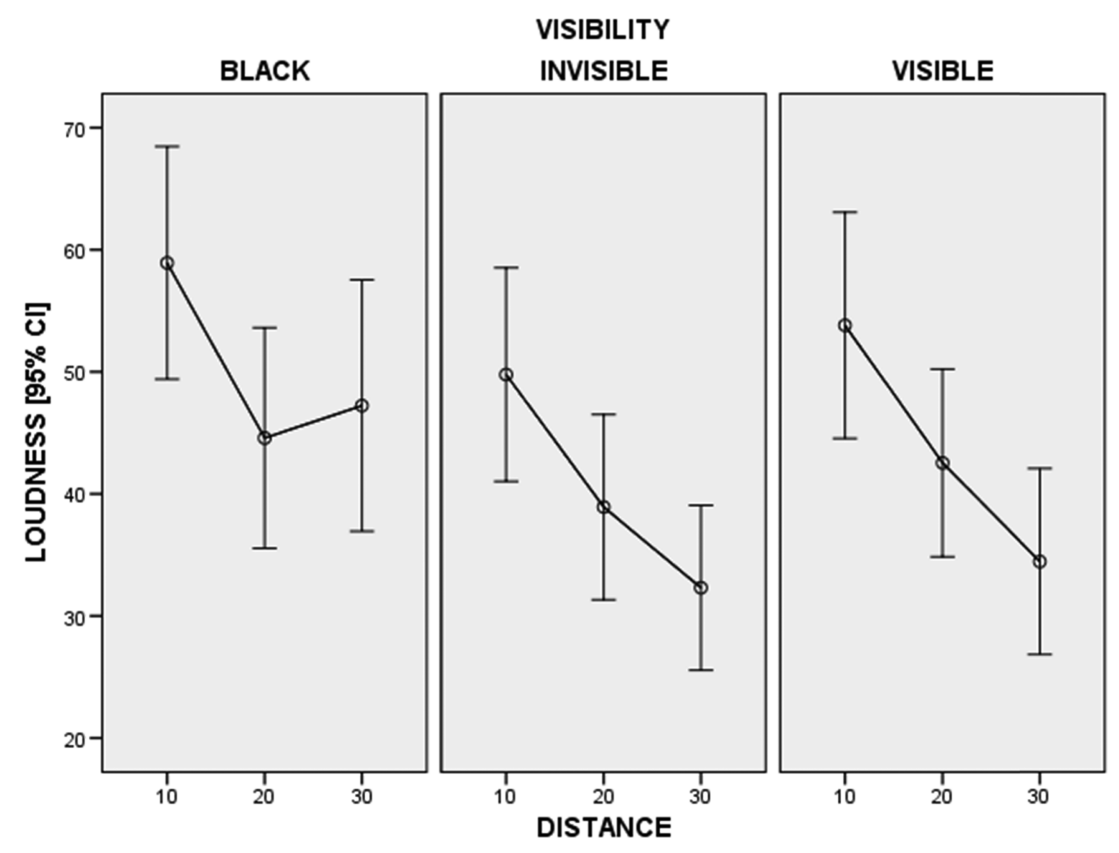

Fig. 7-Perceived loudness estimated marginal means for the visibility levels, as a function of distance.

Ratings of noise annoyance were significantly influenced by the distance factor, $F(2,24)=21.351, p<.001$, partial $\eta^{2}=1.000$, whereas no significant effect was found for the visibility factor or its interaction with the distance. Post hoc analyses revealed that all differences in the noise annoyance ratings for the distance factor were significant. More specifically, the 10-meter scenarios were considered to be more annoying $(M=51.897)$ than the 20-meter scenarios $(M=41.987)$, that were in turn considered to be more annoying than the 30-meter scenarios $(M=38.038)$. Figure 9 reports the estimated marginal means for the annoyance variable, comparing the visibility levels, as a function of distance. Figure 10 shows the individual noise annoyance ratings for the visibility levels, as a function of distance.

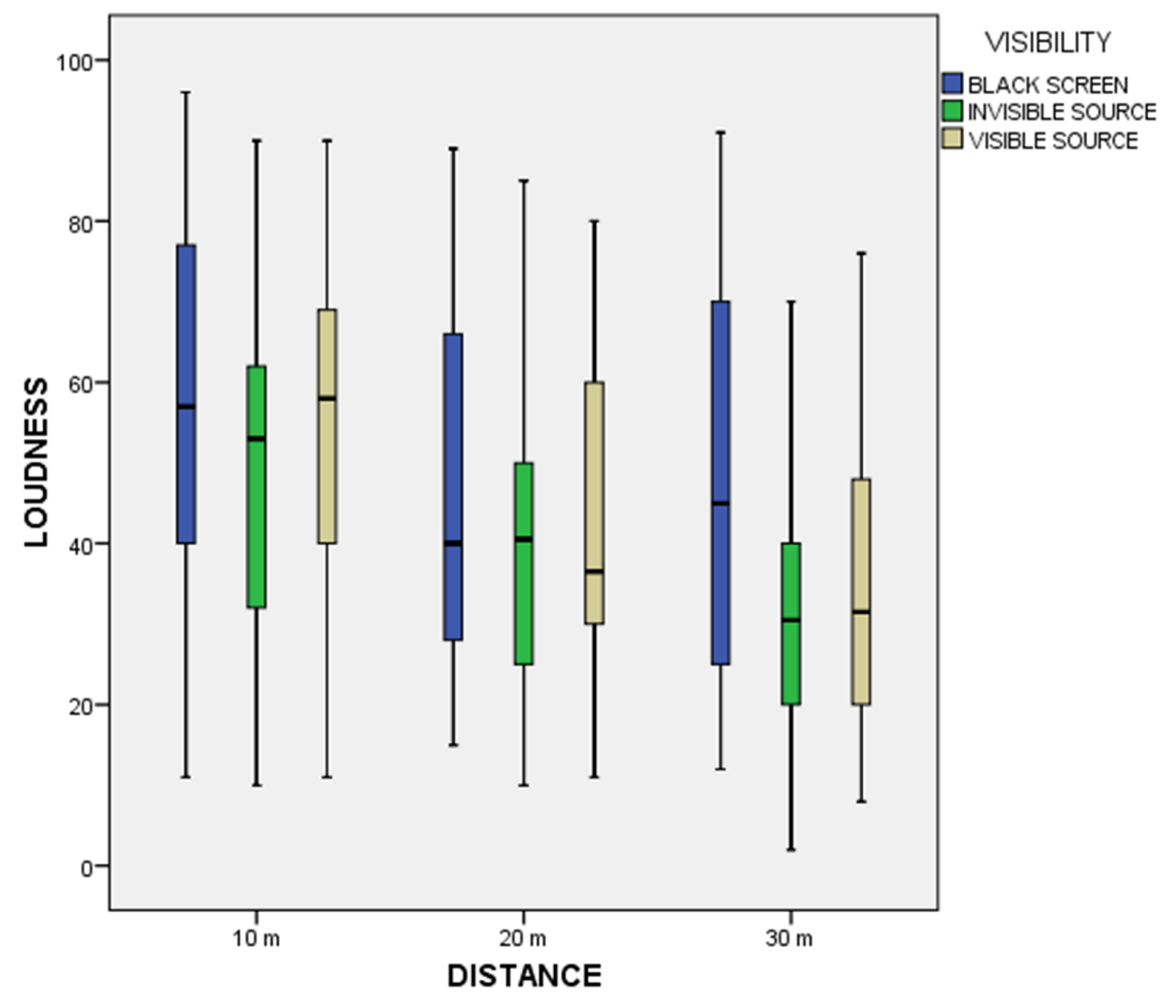

Fig. 8-Perceived loudness assessments for the visibility levels, as a function of distance. 


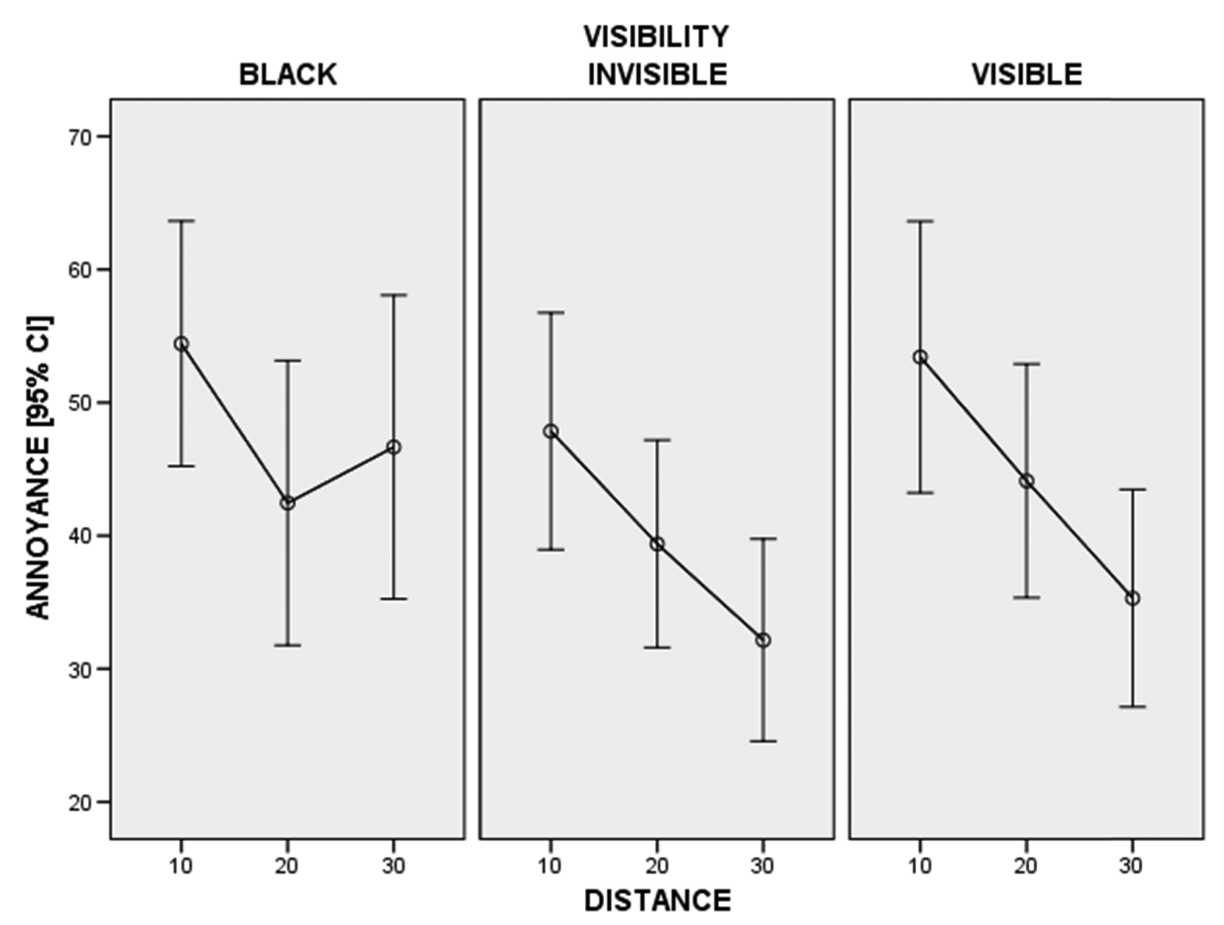

Fig. 9-Noise annoyance estimated marginal means for the visibility levels, as a function of distance.

Additionally, in order to provide further insights on the effect of distance on overall perception, the individual responses to the question related to the visual unpleasantness of the sound source were also analyzed, considering the three scenarios (out of the nine) where the chiller was visible. A one-way repeated measures ANOVA was conducted to evaluate the null hypothesis that there is no effect of distance of the chiller on the individual appraisal of its visual unpleasantness. The results of the ANOVA showed a significant distance effect: Wilks' Lambda $=.744$, $F(2,24)=4.128, p=.029, \eta^{2}=.673$. Therefore, there is significant statistical evidence to reject the null hypothesis

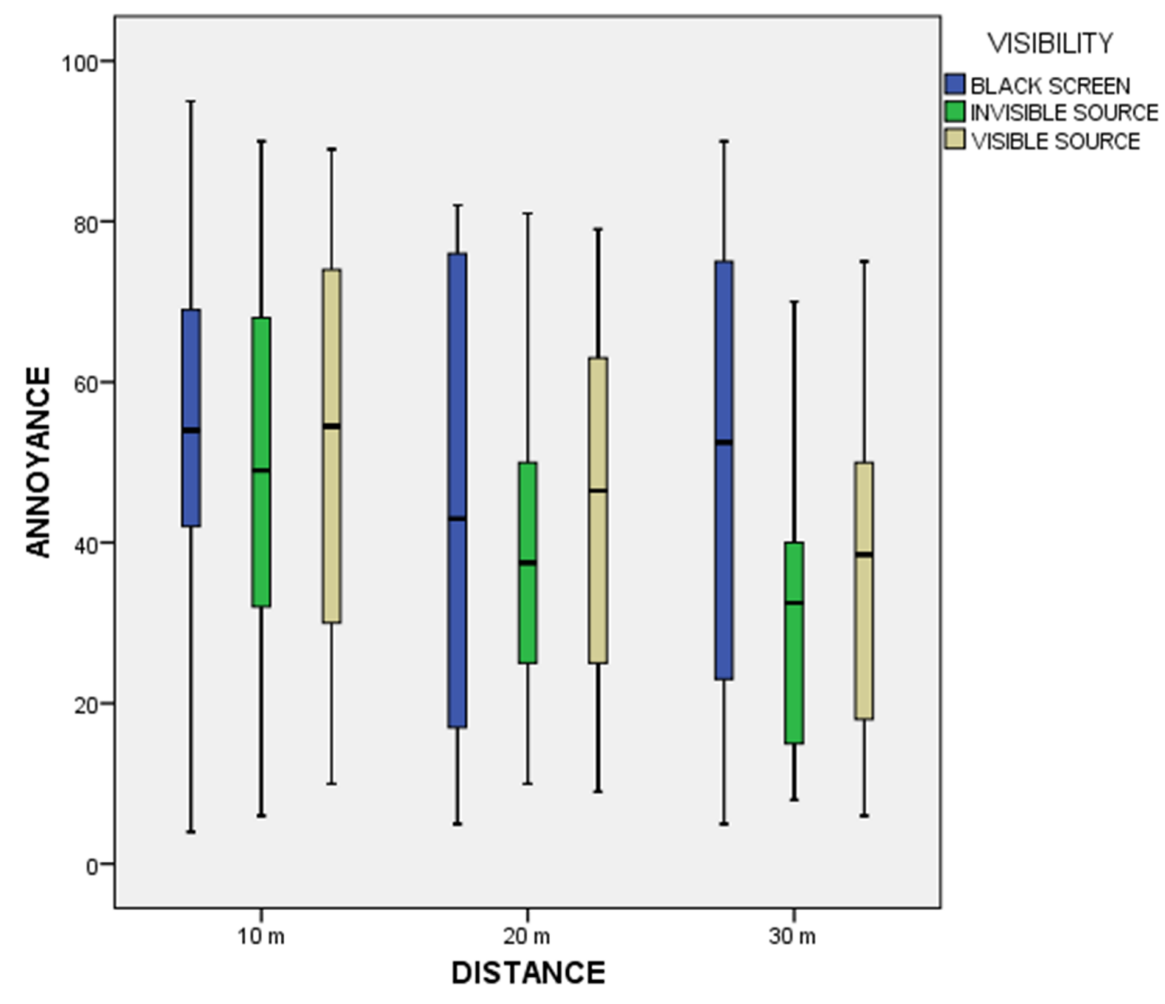

Fig. 10-Noise annoyance assessments for the visibility levels, as a function of distance. 
for the considered variable. However, follow up comparisons showed that not every pairwise difference was significant. The 10-meter scenario differed significantly from the 30-meter scenario $(p=.030)$ and post hoc analysis revealed that the 10-meter scenario was considered to be the most visually unpleasant option $(M=49.65)$ with respect to the 20 meter $(M=42.77)$ and 30-meter scenarios $(M=33.61)$, as shown in Fig. 11 .

\subsection{Experiment 2: Perceived Loudness, Noise Annoyance and Visual Unpleasantness with Cognitive Task}

Similarly as per Experiment 1, a two-way repeated measures ANOVA was carried out to investigate the main effects and interactions of the distance and the visibility factors on the perceived loudness and the noise annoyance variable for Experiment 2.

No significant effect of the visibility factor was observed on perceived loudness. On the other hand, the distance factor had a significant effect, $F(2,24)=5.917$, $p=.002$, partial $\eta^{2}=.955$, as did the interaction between visibility and distance, $F(2,24)=8.304, p=.002$, partial $\eta^{2}=.938$. Post hoc analyses revealed that the 30 -meter scenarios were considered significantly quieter $(M=34.808)$ than both the 10-meter scenarios $(M=45.205)$ and the 20-meter scenarios $(M=44.744)$, but 10 - and 20-meter scenarios did not differ significantly. Figure 12 reports the estimated marginal means for the loudness variable, comparing the visibility levels, as a function of distance, for Experiment 2. Figure 13 shows the individual assessments of perceived loudness for the visibility levels, as a function of distance, for Experiment 2.

Regarding the noise annoyance, only the distance factor produced a significant effect, $F(2,24)=4.560$, $p=.021$, partial $\eta^{2}=.719$. Post hoc analyses revealed that only the differences between the 20-meter scenarios $(M=40.333)$ and the 30-meter scenarios $(M=35.821)$ were significant, while this did not occur with respect to the 10 -meter scenarios $(M=41.423)$. Figure 14 reports the estimated marginal means for the annoyance variable, comparing the visibility levels, as a function of distance, for Experiment 2. Figure 15 shows the individual noise annoyance ratings for the visibility levels, as a function of distance, for Experiment 2.

Similarly, as per Experiment 1, the individual responses to the question related to the visual unpleasantness of the sound source were analyzed, considering the three scenarios where the chiller was visible, while participants were performing the cognitive task. A one-way repeated measures ANOVA was conducted to evaluate the null hypothesis that there is no effect of distance of the chiller on the individual appraisal of its visual unpleasantness. The results of the ANOVA showed also in the case of the cognitive task a significant distance effect: Wilks' Lambda $=.526, F(2,24)=10.797, p<.001, \eta^{2}=$ .980. Therefore, there is significant statistical evidence to reject the null hypothesis for the considered variable. The

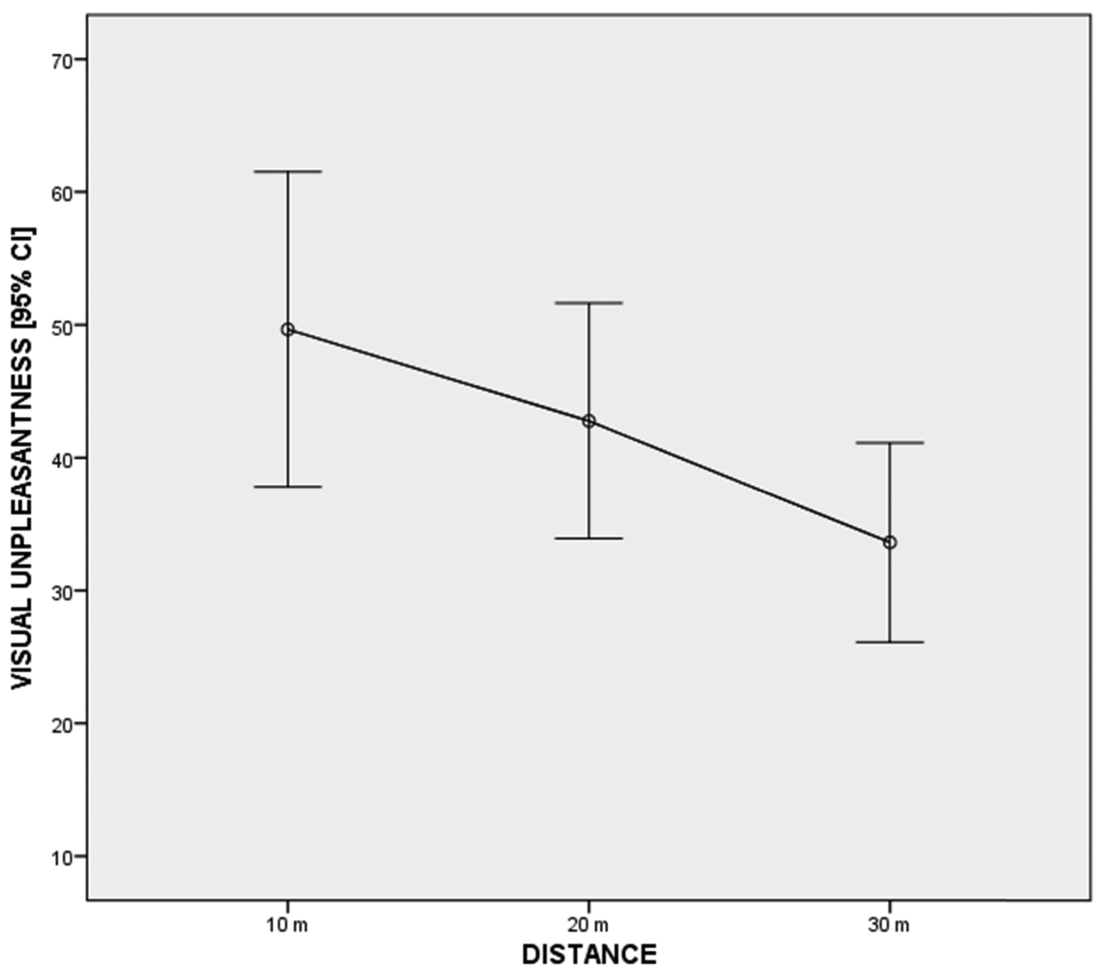

Fig. 11-Visual unpleasantness estimated marginal means as a function of distance. 


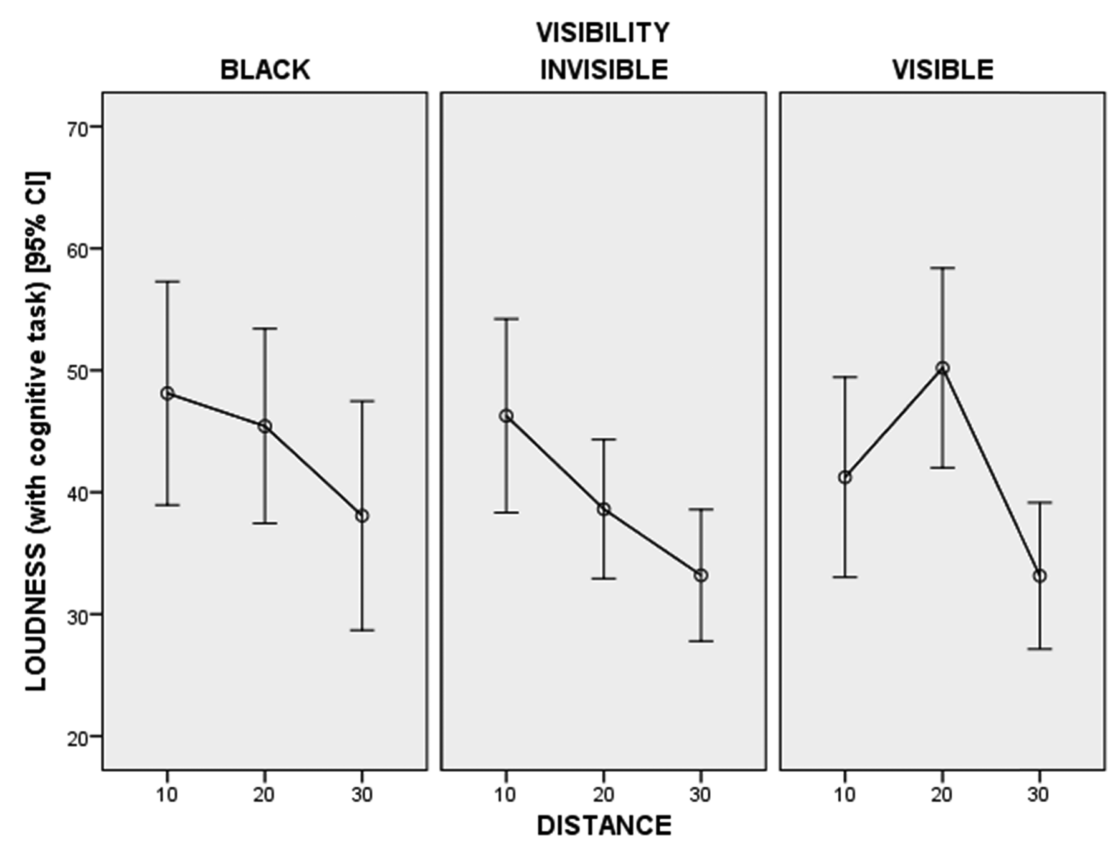

Fig. 12-Perceived loudness estimated marginal means for the visibility levels, as a function of distance, in the case of participants performing a cognitive task.

30-meter scenario differed significantly both from the 10 -meter scenario $(p=.001)$ and the 20-meter scenario $(p=.008)$. Post hoc analysis revealed that the 10-meter scenario was considered to be the most visually unpleasant option $(M=36.61)$ with respect to the 20-meter $(M=33.65)$ and 30-meter scenarios $(M=21.77)$, as shown in Fig. 16.

\section{DISCUSSION}

Within the present research, Experiment 1 was meant to test the effect of the visibility of the sound source (i.e. the chiller) on its perceived loudness and related noise annoyance, as assessed by individuals, also taking into account

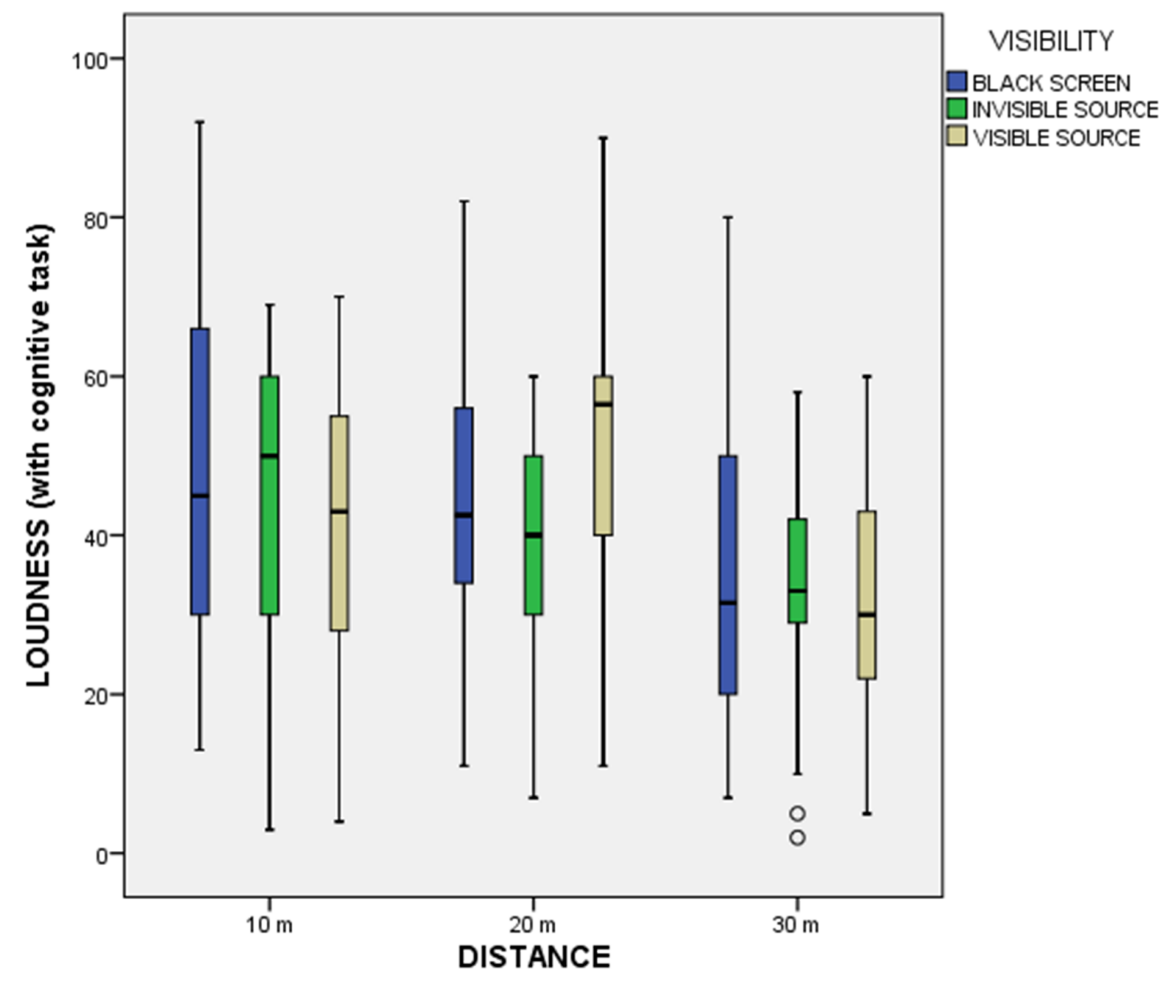

Fig. 13-Perceived loudness assessments for the visibility levels, as a function of distance, in the case of participants performing a cognitive task. 


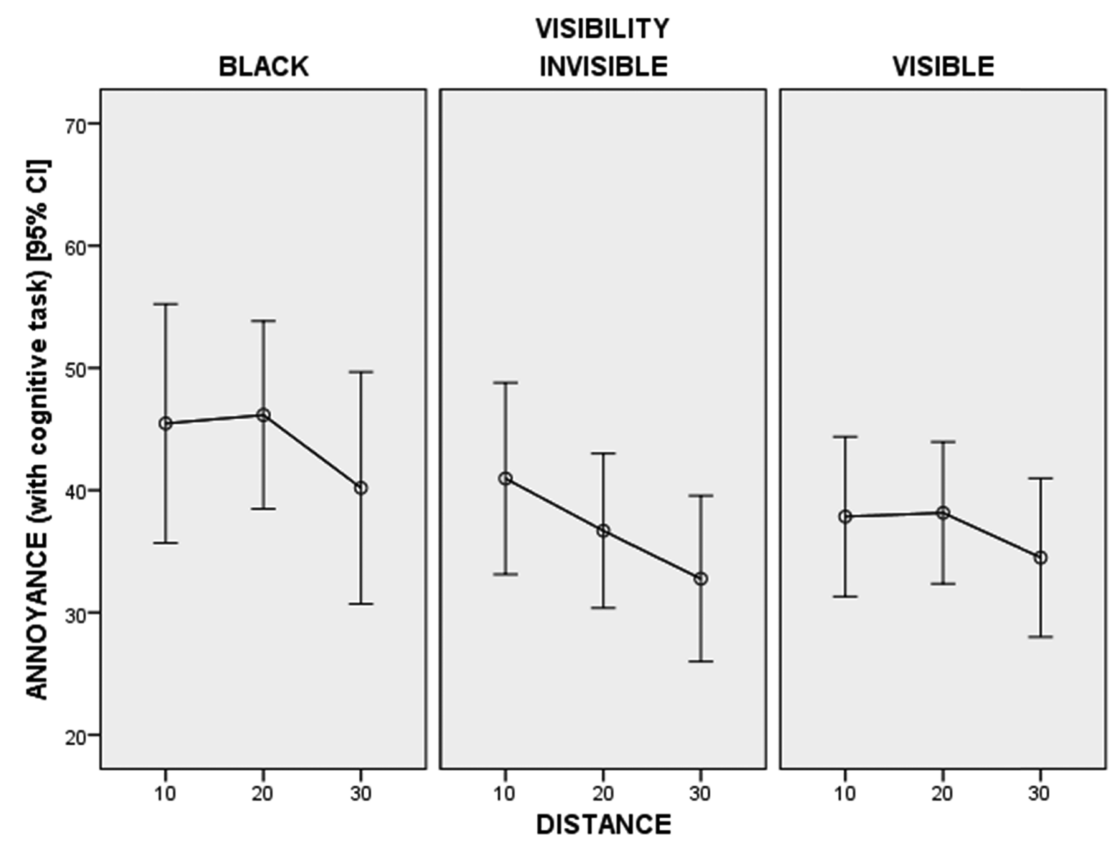

Fig. 14-Noise annoyance estimated marginal means for the visibility levels, as a function of distance, in the case of participants performing a cognitive task.

distance as a factor. Results from Experiment 1 show that both perceived loudness and self-reported noise annoyance were modified by the distance factor. Such a finding was expected, as in this case the individual perception of noise reflects the physical decrease of the stimulus' auditory intensity; that is, the farther the source, the quieter the noise, the smaller the perceived loudness and the corresponding noise annoyance.

However, the results for the visibility factor point out a different dimension of the sound source's assessment. Regarding the perceived loudness ratings, the scenario with the black screen differed significantly from both

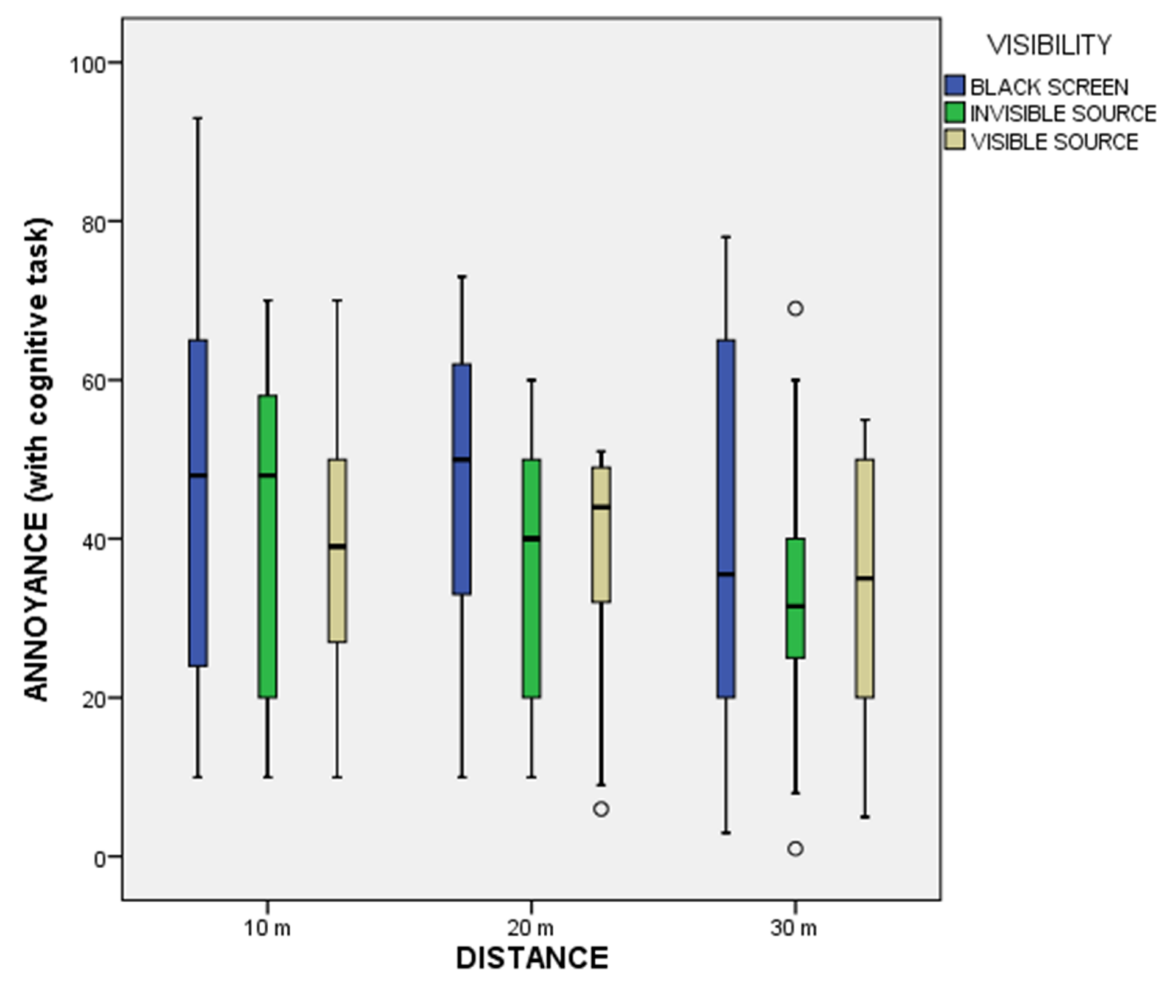

Fig. 15-Noise annoyance assessments for the visibility levels, as a function of distance, in the case of participants performing a cognitive task. 


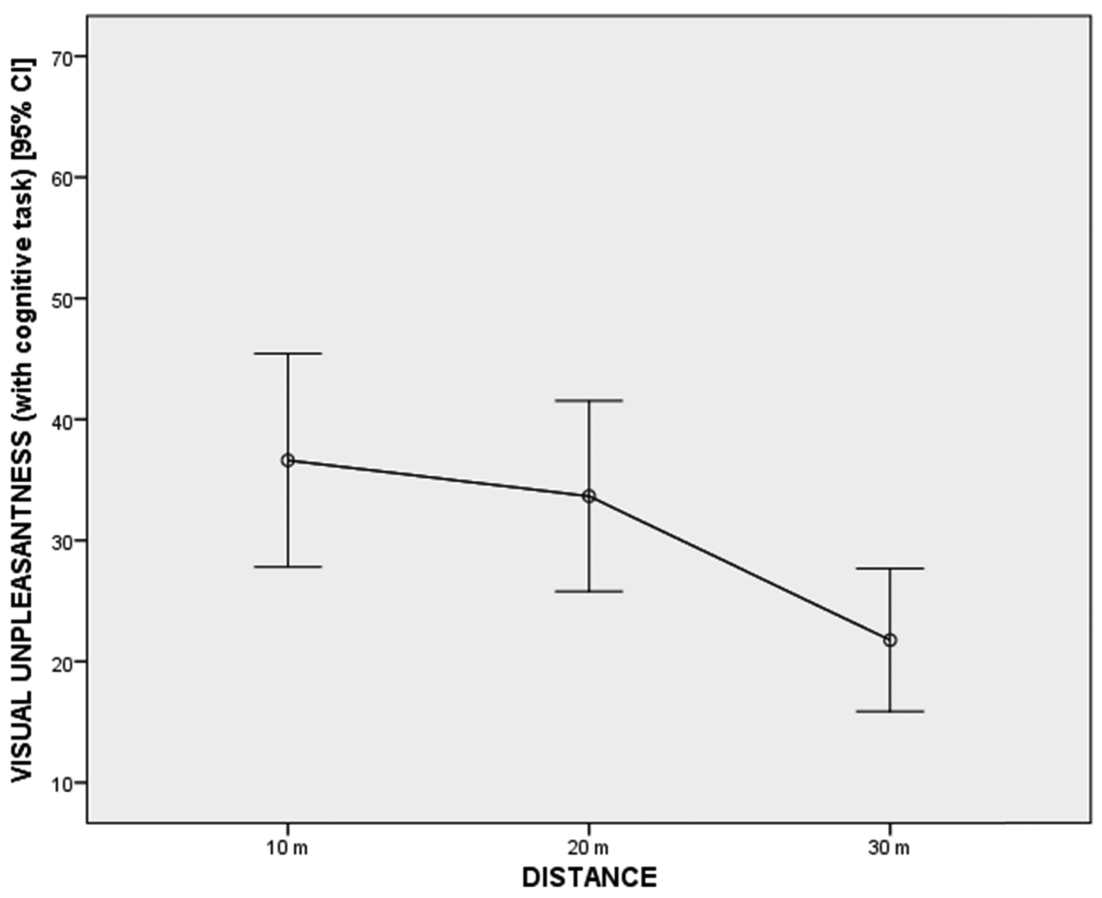

Fig. 16-Visual unpleasantness estimated marginal means as a function of distance, in the case of participants performing a cognitive task.

the invisible source and the visible source scenarios, but the invisible and visible sources did not differ significantly between them. A possible explanation for this could be the different individual interpretation of the auditory stimulus location relative to the body ${ }^{49}$. When the participants have a visual reference space, the source producing the noise they experience in the IVR scenarios is located in the "extrapersonal" space, either because it is reasonably far (i.e. 10, 20 and $30 \mathrm{~m}$ accordingly, for the visible source scenarios) or because it is not visible at all (i.e. for the invisible source scenarios). The perceived loudness is consistent, regardless of the sound source being visible or not. On the other hand, when the participants have no visual reference space (i.e. black screen scenarios), the
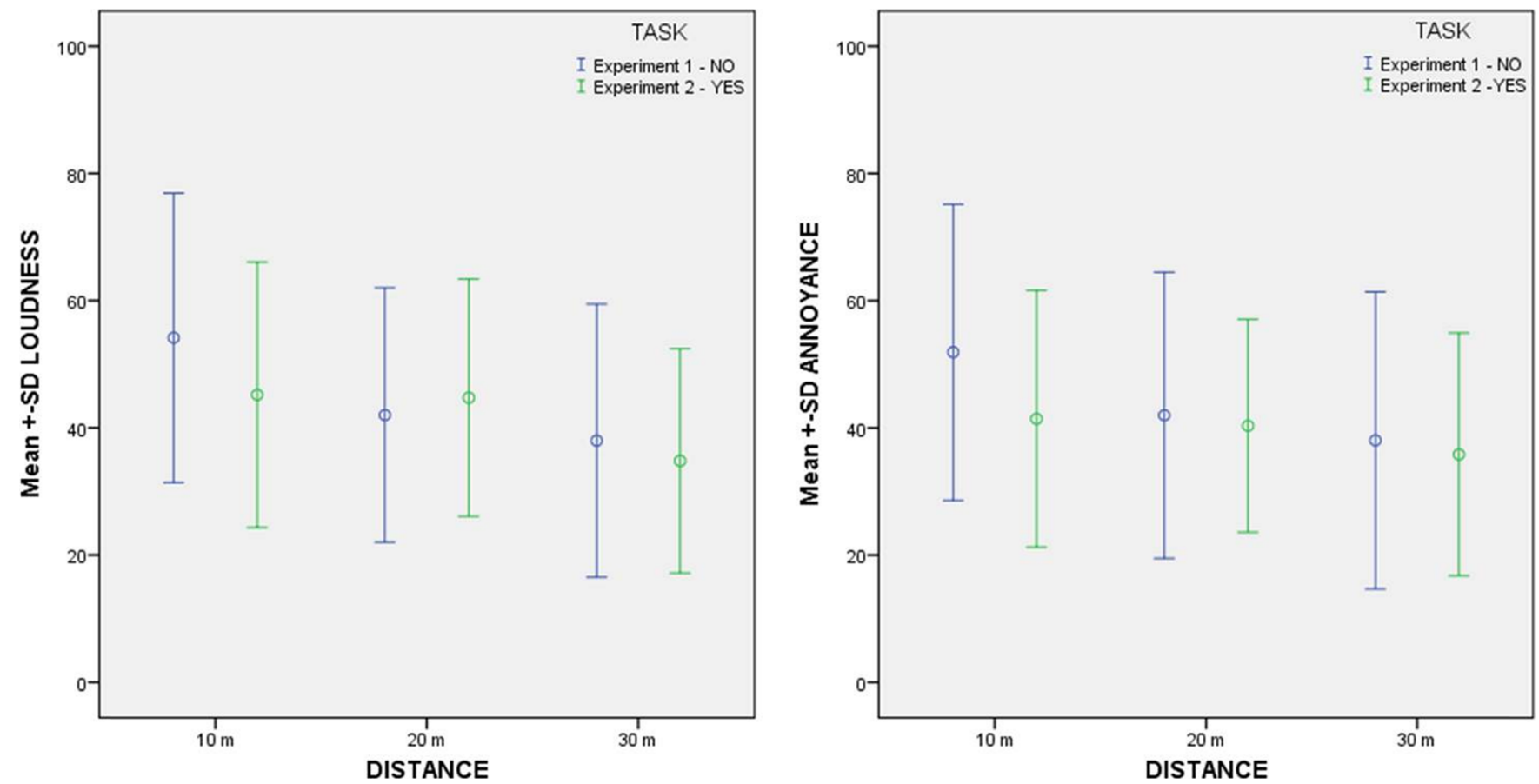

Fig. 17-Comparison between the estimated marginal means for the perceived loudness (left) and noise annoyance (right) variables of the experiments 1 and 2. 
sound source is possibly perceived to be closer, and therefore louder, like it belonged to the "peripersonal" space of the subject. Overall, the presence of contextual visual information associated with the chiller sound affected the noise individual assessment. This is consistent with previous findings in literature, showing that the processing of the auditory information is holistic and relies also on factors other than acoustic ones ${ }^{50,51}$.

Even though a similar pattern would have been expected for the self-reported noise annoyance, no significant differences were observed for the visibility factor in that variable. This raises a question about possible preconceptions that participants might likely have towards a sound source that they consider as unwanted, that is, not pleasant. The results of the one-way repeated measures ANOVA for the visual unpleasantness variable seem to support this effect, as the visual unpleasantness of the sound source linearly decreases with distance, showing that the sample has a negative attitude towards the chiller itself and prefers when it is far.

The rationale for performing Experiment 2 was testing whether the presence of a "distractor" (i.e. the cognitive task) could affect the perceived loudness and noise annoyance ratings. Participants were required to count backward, starting from a random number, while experiencing the audio-visual scenarios and then answer the same questions as per Experiment 1. The experimenter took note of the number of answers correctly given by the participants, but that information was not considered within the present study, inasmuch as the assumption was that, for this particular sound source, the cognitive task was affecting the noise assessment, rather than the noise affecting the cognitive performance. Significant differences were observed in both perceived loudness and noise annoyance ratings for the distance factor, showing that the participants were still able to discriminate the different levels of the auditory stimuli. Conversely, no significant effect of the visibility factor was observed on perceived loudness or noise annoyance, suggesting that individuals tended to discard the visual information in the presence of a task requiring executive control.

Comparing the estimated marginal means for the perceived loudness and noise annoyance ratings between Experiment 1 and Experiment 2, it can be observed that the cognitive task, overall, had a mitigation effect on the auditory stimuli, as shown in Fig. 17.

As reported in Introduction section, the effect of source visibility on noise perception is not uniquely determined. People reacted differently to different sound sources. From an auditory point of view, this could be due to different sound levels, as well as spectral or temporal characteristics of the sound sources. In general, for road traffic noise with relatively high sound levels (i.e. approximately $70 \mathrm{~dB}$ ), visibility has a deteriorating effect (i.e. increase of perceived loudness and/or noise annoyance $)^{35,38}$. On the other hand, considering railway noise with similar sound levels, visibility was found to decrease perceived loudness and noise annoyance $^{36}$; thus, when the sound source is not as constant as road traffic, people might prefer to see it. However, when sound levels significantly decrease (i.e. approximately $40 \mathrm{~dB}$ ) the differences between visible and non-visible sound source stimuli become weak or negligible ${ }^{34}$. At such low levels, other non-acoustical factors might be more relevant. Vision-related aspects other than visibility of the source (e.g. colour, size of the sound source) and overall complexity of the visual scenario ${ }^{52}$ are likely to affect noise perception in such conditions. Furthermore, individual factors like familiarity or preconception (e.g. general attitude towards the source or aesthetic preference) can also play an important role in the perceptual process when sound levels drop ${ }^{53}$.

Within the framework of this research, participants were overall rather negative towards the chiller, which is confirmed by the visual unpleasantness data. Taken together, the two experiments of this study tell us that when a reference visual context is given, like in the situation of a living room environment, the visibility of the chiller producing the noise is not relevant for its assessment in terms of loudness and annoyance. The sound source used for this study had low levels (36-46 dB) and was characterized by small spectral and temporal variation, so the lack of effect would be in line with Aylor and Marks ${ }^{34}$. Pedersen and Larsman ${ }^{9}$, in their study on wind turbines, showed that the individual self-reported noise annoyance was higher when the noise source was visible, so this would be in contrast with this study's findings, since sound levels were rather low in their case. Yet, wind turbine noise has a very specific temporal and spectral pattern which is likely to make it more noticeable to the human ear. The authors also pointed out that the visual contrast between the technological source (i.e. the wind turbine) and an essentially rural background might play a role in the annoyance assessment, while in the present study the visual scenario was relatively complex (living room with window facing a peri-urban context) and the source was associated with no motion, so it seems likely to assume that people's attention was more broadly distributed, limiting the source visibility effect.

\section{CONCLUSIONS}

This paper investigated the influence of the visibility and the distance of a chiller from an observer in an ordinary living environment on the perceived loudness and annoyance connected to its noise. Two separate experiments were conducted with the same sample of participants in an immersive virtual reality environment to test the same experimental design with and without a cognitive task. Future experiments to extend the participants' 
sample composition might be desirable to better generalize the observed results.

Assuming that the presented results should be limited to the investigated sound source (i.e. a chiller unit) with a relatively low level and small temporal variation, the main conclusions of this study are as follows:

- The distance of the chiller, corresponding to different actual sound levels, influenced the perceived loudness of its noise and the related noise annoyance, despite the visual factor; the individual mean differences were $M_{10 \mathrm{~m}-20 \mathrm{~m}}=$ 12.15 and $M_{20 \mathrm{~m}-30 \mathrm{~m}} 4.01$ for the perceived loudness and $M_{10 \mathrm{~m}-20 \mathrm{~m}}=9.91$ and $M_{20 \mathrm{~m}-30 \mathrm{~m}}$ 3.95 for the noise annoyance.

- When a visual reference context was provided, the visibility of the chiller itself did not affect the perceived loudness and related noise annoyance; that is, the scenarios with black screen differed from both the visible source and invisible source scenarios, but there were no significant differences between the scenarios with a visual stimulus (regardless of the sound source being visible or not).

- Performing a cognitive task overall reduced the mean individual ratings of both the perceived loudness and noise annoyance related to the chiller, suggesting that for the investigated sound source, characterized by small spectral and temporal variation, executive control processes might inhibit attentional listening.

The above findings suggest that, for these kinds of chillers, which are becoming increasingly common in the urban realm, the visibility of the source is not a significant influencing factor for noise perception, although for other sources (e.g. traffic noise), previous studies did report that visibility might have an influence in this regard. This result suggests that individuals' preconception towards particular noise sources is a relevant component of environmental sound sources' perception, and this is supported by the fact that the mean visual unpleasantness decreases with the increasing distance of the chiller. While the effect of external view is limited, designers and planners could carefully consider functional design of the indoor spaces, for example, exposing the living and working spaces to chiller noise, rather than bedrooms and quiet sides, which could compensate to some extent the annoyance connected with such noise sources.

\section{ACKNOWLEDGEMENTS}

This research received funding through the People Programme (Marie Curie Actions) of the European
Union's 7th Framework Programme FP7/2007-2013 under REA grant agreement $n^{\circ}$ 290110, SONORUS “Urban Sound Planner." The authors are also grateful to Paola Simeoni for providing support during the audio recordings and to Jim Uttley for his comments on the paper.

\section{APPENDIX}

This is an adapted version of the instructions read to the participants for Experiment 1 and Experiment 2 at the beginning of the session [original version in Italian].

\subsection{Instructions for Experiment 1}

Dear Participant,

Thank you for taking part in this experiment. We aim to investigate how people perceive the sound produced by chillers and/or air-conditioning systems. To this purpose, we will ask you to experience a number of audiovisual scenarios by means of immersive virtual reality devices and answer some questions for each scenario.

In most of cases the virtual scenario will represent you sitting in a living room with an open window. You will listen to the sound produced by a chiller on the roof of a building outside to room. Sometimes you will be able to see the chiller outside the room, sometimes you will not. Sometimes there will be no visual scenario at all (you will see a black screen through your visor), but the sound will still be there. In any case, when the scenario is launched, please look in front of you and listen.

After each scenario, you will be asked to answer two questions by dragging a cursor with the mouse, using the laptop we provide. When a scenario where the chiller is visible is presented, an additional question will be asked.

Before we start, we are going to present you a test scenario so that you can familiarize with the virtual environment. This is not part of the experiment and no question will be asked. As soon as you are ready, please let us know.

\subsection{Instructions for Experiment 2}

Dear Participant,

Thank you for taking part in this experiment. We aim to investigate how people perceive the sound produced by chillers and/or air-conditioning systems. To this purpose, we will ask you to experience a number of audiovisual scenarios by means of immersive virtual reality devices and perform a simple task, and answer some questions for each scenario.

In most of cases the virtual scenario will represent you sitting in a living room with an open window. You will listen to the sound produced by a chiller on the roof of a building outside to room. Sometimes you will be able to see the chiller outside the room, sometimes you will not. 
Sometimes there will be no visual scenario at all (you will see a black screen through your visor), but the sound will still be there. In any case, when the scenario is launched, please look in front of you and listen. As soon as the scenario is launched, a random two-digit number will appear on the screen of your visor. When this happens, please start counting loud, backward in steps of seven, starting from the number you read (e.g., if you read " 58 ", you should start counting loud: " $51,44,37 .$. " and so on).

After each scenario, you will be asked to answer two questions by dragging a cursor with the mouse, using the laptop we provide. When a scenario where the chiller is visible is presented, an additional question will be asked.

Before we start, we are going to present you a test scenario so that you can familiarize with the virtual environment. This is not part of the experiment and no question will be asked. As soon as you are ready, please let us know.

\section{REFERENCES}

1. World Health Organization, Burden of Disease from Environmental Noise, WHO Regional Office for Europe, Copenhagen, Denmark, (2011).

2. E. Pedersen, "City dweller responses to multiple stressors intruding into their homes: Noise, light, odour, and vibration", Int. J. Environ. Res. Public Health, 12(3), 3246-3263, (2015).

3. R. Guski, R. Felscher-Suhr and R. Schuemer, "The concept of noise annoyance: How international experts see it", J. Sound Vibr., 223(4), 513-527, (1999).

4. R.F. Job, "Community response to noise: A review of factors influencing the relationship between noise exposure and reaction", J. Acoust. Soc. Am., 83(3), 991-1001, (1988).

5. J.M. Fields, "Effects of personal and situational variables on noise annoyance in residential areas", J. Acoust. Soc. Am., 93(5), 2753-2763, (1993).

6. $\overline{H . M}$. Miedema and H. Vos, "Exposure-response relationships for transportation noise", J. Acoust. Soc. Am., 104(6), 3432-3445, (1998).

7. H.M. Miedema and C.G. Oudshoom, "Annoyance from transportation noise: Relationships with exposure metrics DNL and DENL and their confidence intervals", Environ. Health Perspect, 109(4), 409-416, (2001).

8. E. Pedersen and K. Persson Waye, "Wind turbine noise, annoyance and self-reported health and well-being in different living environments", J. Occup. Env. Med., 64(7), 480-486, (2007).

9. E. Pedersen and P. Larsman, "The impact of visual factor on noise annoyance among people living in the vicinity of wind turbines", J. Environ. Psychol., 28(4), 379-389, (2008).

10. J.A. Ballas, "Common factors in the identification of an assortment of brief everyday sounds", J. Environ. Psychol., 19(2), 250-267 (1993).

11. J.A. Paulauskis, "Addressing noise problems in screw chillers", ASHRAE J, 22-25, (1999).

12. S.B. Knight, J.B. Evans and C.N. Himmel, "Case study: Air cooled chillers with rotary helical (screw) compressors at hospital with impact on patient rooms, residential neighborhoods, and open park", InterNoise01, (2001).

13. B.T. Wang, C.H. Hsieh, W.C. Wang and C.L. Liu, "Noise and vibration characteristic studies of twin screw compressor in different operating conditions", International Compressor Engineering Conference, (2012).
14. J.B. Evans, C.N. Himmel and J.D. Leasure, "Environmental noise case studies: Air-cooled refrigeration chiller installations near residential structures", ASHRAE Trans., 118(2), 50-57, (2012).

15. M.J. Crocker, J.P. Arenas and R.E. Dyamannavar, "Identification of noise sources on a residential split-system air-conditioner using sound intensity measurements", Appl. Acoust., 65(5), 545-558, (2004).

16. K. Persson Waye and R. Rylander, "Disturbance from lowfrequency noise in the environment: A survey among the local environmental health authorities in Sweden", J. Sound Vibr., 121(2), 339-345, (1988).

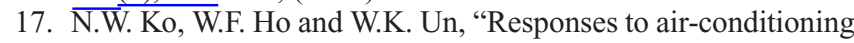
system noise", J. Sound Vibr., 57(4), 595-602, (1978).

18. R. Sottek and K. Genuit, "Sound quality evaluation of fan noise based on advanced hearing-related parameters", Noise Control Engr. J., 57(4), 384-390, (2009).

19. M. Alayrac, C. Marquis-Favre, S. Viollon, J. Morel and G. Le Nost, "Annoyance from industrial noise: Indicators for a wide variety of industrial sources", J. Acoust. Soc. Am., 128(3), 1128-1139, (2010).

20. M. Alayrac, C. Marquis-Favre and S. Viollon, "Total annoyance from an industrial noise source with a main spectral component combined with a background noise", J. Acoust. Soc. Am., 130(1), 189-199, (2011).

21. A. Preis, J. Kociński, H. Hafke-Dys and M. Wrzosek, "Audiovisual interactions in environment assessment", Sci. Total Environ., 523, 191-200, (2015).

22. H. McGurk and J. MacDonald, "Hearing lips and seeing voices", Nature, 264, 746-748, (1976).

23. D.H. Warren, T.J. McCarthy and R.B. Welch, "Discrepancy and non-discrepancy methods of assessing visual-auditory interaction”, Atten. Percept. Psychophys., 33(5), 413-419, (1983).

24. F. Frassinetti, N. Bolognini and E. Ladavas, "Enhancement of visual perception by crossmodal visuo-auditory interaction", Exp. Brain Res., 147(3), 332-343, (2002).

25. M.O. Ernst and B.B. Bülthoff, "Merging the senses into a robust percept", Trends Cogn. Sci., 8(4), 162-169, (2004).

26. S. Watkins, L. Shams, O. Josephs and G. Rees, "Activity in human V1 follows multisensory perception", Neuroimage, 37(2), 572-578, (2007).

27. B.L. Allman and M.A. Meredith, "Multisensory processing in 'unimodal' neurons: Cross-modal subthreshold auditory effects in cat extrastriate visual cortex", J. Neurophys., 98(1), 545-549, (2007).

28. L. Shams and R. Kim, "Crossmodal influences on visual perception”, Phys. Life Rev., 7(3), 269-284, (2010).

29. T. Iachini, L. Maffei, F. Ruotolo, V.P. Senese, G. Ruggiero, M. Masullo and N. Alekseeva, "Multisensory assessment of acoustic comfort aboard metros: A virtual reality study", Appl. Cognitive Psych., 26(5), 757-767, (2012).

30. F. Ruotolo, V.P. Senese, G. Ruggiero, L. Maffei, M. Masullo and T. Iachini, "Individual reactions to a multisensory immersive virtual environment: The impact of a wind farm on individuals", Cogn. Process., 13(Supp. 1), 319-323, (2012).

31. S. Viollon, C. Lavandier and C. Drake, "Influence of visual setting on sound ratings in an urban environment", Appl. Acoust., 63(5), 493-511, (2002).

32. R.J. Pheasant, K.V. Horoshenkov and G.R. Watts, "The role of audio-visual interaction on the perception of tranquility", Euronoise09, (2009).

33. D. Menzel, H. Fastl, R. Graf and J. Hellbruck, "Influence of vehicle colors on loudness judgment", J. Acoust. Soc. Am., 123(5), 2477-2479, (2008).

34. D.E. Aylor and L.E. Marks, "Perception of noise transmitted through barriers", J. Acoust. Soc. Am., 59(2), 397-400, (1976).

35. J.L. Joynt and J. Kang, "The influence of preconceptions on perceived sound reduction by environmental noise barriers", Sci. Total Environ., 408(20), 4368-4375, (2010). 
36. L. Maffei, M. Masullo, F. Aletta and M. Di Gabriele, "The influence of visual characteristics of barriers on railway noise perception”, Sci. Total Environ., 445-446, 41-47, (2013).

37. E. Peris, J. Woodcock, G. Sica, C. Sharp, A.T. Moorhouse and D.C. Waddington, "Effect of situational, attitudinal and demographic factors on railway vibration annoyance in residential areas", J. Acoust. Soc. Am., 135(1), 194-204, (2014).

38. Z. Bangjun, S. Lili and D. Guoqing, "The influence of the visibility of the source on the subjective annoyance due to its noise", Appl. Acoust., 64(12), 1205-1215, (2003).

39. F. Faul, E. Erdfelder, A.G. Lang and A. Buchner, "G*Power 3: A flexible statistical power analysis program for the social, behavioral, and biomedical sciences", Behav Res. Methods, 39(2), 175-191, (2007).

40. J. Cohen, Statistical Power Analysis for the Behavioral Sciences, Lawrence Erlbaum Associates, Hillsdale, New Jersey, (1988).

41. "Acoustics-Attenuation of sound during propagation outdoors. Part 2: General method of calculations", International Standard ISO 9613-2: 1996, International Organization for Standardization, (1996).

42. X. Wang, M.J. Kim, P.E. Love and S.C. Kang, "Augmented reality in built environment: Classification and implications for future research", Automat. Constr., 32, 1-13, (2013).

43. L. Maffei, M. Masullo, A. Pascale, G. Ruggiero and V. Puyana Romero, "On the validity of immersive virtual reality as tool for multisensory evaluation of urban spaces", Energy Procedia, 78, 471-476, (2015).

44. M. Vorländer, Fundamentals of Acoustics, Modelling, Simulation, Algorithms and Acoustic Virtual Reality, Springer, Aachen, Germany, (2008).
45. A. Lindau and S. Weinzierl, "Assessing the plausibility of virtual acoustic environments", Acta Acust. United with Acustica. 98(5), 804-810, (2012).

46. R.S. Pellegrini, A Virtual Reference Listening Room as an Application of Auditory Virtual Environments, Ruhr Universität Bochum, Bochum, Germany, (2001).

47. M. Slater, "Place illusion and plausibility can lead to realistic behaviour in immersive virtual environments", Philos. Trans. R. Soc. Lond., B, Biol. Sci., 364, 3549-3557, (2009).

48. F. Ruotolo, L. Maffei, M. Di Gabriele, T. Iachini, M. Masullo, G. Ruggiero and V.P. Senese, "Immersive virtual reality and environmental noise assessment: An innovative audio-visual approach", Environ. Impact Assess., 41, 10-20, (2013).

49. N.P. Holmes and C. Spence, "The body schema and multisensory representation(s) of peripersonal space", Cogn. Process., 5(2), 94-105, (2004).

50. R.J. Pheasant, M.N. Fisher, G.R. Watts, D.J. Whitaker and K.V. Horoshenkov, "The importance of auditory-visual interaction in the construction of "tranquil space"', J. Environ. Psychol., 30(4), 501-509, (2010).

51. W.J. Davies, N.S. Bruce and J.E. Murphy, "Soundscape reproduction and synthesis", Acta Acust. united with Acustica., 100(2), 285-292, (2014).

52. L. Maffei, T. Iachini, M. Masullo, F. Aletta, F. Sorrentino, V.P. Senese and F. Ruotolo, "The effects of vision-related aspects on noise perception of wind turbines in quiet areas", Int. J. Environ. Res. Public Health, 10(5), 1681-1697, (2013).

53. J.Y. Hong and J.Y. Jeon, "The effects of audio-visual factors on perceptions of environmental noise barrier performance", Landsc. Urban Plan., 125, 28-37, (2014). 